\title{
1916 : An 1 du dentiste militaire en France
}

\section{6: The year when military dentistry was created} in Fronce

MOTS-CLEFS :
- Chirurgie dentaire,
histoire, Première Guerre
mondiale, service de
santé des armées
françaises.

\section{KEYWORDS:}

- Dental surgery, history, World Wor I, French Military Health Service.
AOS 2014;268:16-23

DOI: $10.1051 / \mathrm{aos} / 2014203$

(C) EDP Sciences 2014
Résumé

La fin du XIX ${ }^{\mathrm{e}}$ siècle a été une période faste pendant laquelle la chirurgie dentaire française a connu de grandes avancées techniques et structurelles : création des premières écoles dentaires (1880; 1884), loi Paul Brouardel (1892) qui confère un statut légal à la chirurgie dentaire en imposant une formation suivie au sein d'une faculté de médecine sanctionnée ò la fin par un examen, naissance de l'odontologie médico-légale (1897), etc. C'est donc une profession balbutiante, mais légiférée depuis 1892, ayant acquis de vraies lettres de noblesse, qui entame la guerre en 1914. Les dentistes sont alors cantonnés dans des rôles très subalternes (brancardiers, infirmiers, etc.) où ils multiplient les actions héroïques, sans jamais exercer leur art. Sous la pression de certains journaux, qui s'étonnent de leur absence au sein du service de santé des armées, et de diverses instances professionnelles, avec la mise en place très vite au cours du conflit de services de stomatologie entièrement consacrés à la réparation des " gueules cassées » détruites pendant les combats où les dentistes jouent un rôle capital, Raymond Poincaré, alors président de la République, finit par ordonner, en 1916, la création du dentiste militaire au sein des armées françaises. De fait, pour atteindre pareil accomplissement, quels ont été les acteurs prépondérants qui ont œuvré à cette cause et quels ont été les obstacles qui se sont dressés sur leur route?

\author{
Abstract
}

The end of the $19^{\text {th }}$ century was a prosperous period during which French dental surgery had known significant advanced and structural techniques: the creation of the first dental schools (1880; 1884), the enactment of Paul Brouardel's law (1892) which gave dental surgery a legal status by imposing trainship and a corresponding final examination within a medical university, the birth of forensic dentistry (1897), etc. Therefore, it was a profession still in its infoncy but legislated since 1892 and which had won its spurs that got involved in the war for the first time in 1914. At the time, the dentists were limited to low-ranking roles (hospital porters, nurses, etc.) where they without practicing their dental art. However, during the conflict, services entirely devoted to stomatology were gradually implemented due to the need to repair the "Broken faces " damaged during the fighting where the dentists role was of paramount importance. Some newspapers started to wonder more and more why the practice of dentistry was absent from the Military Health Service and from various professional organisations while its role was obviously of utmost importance during the combats. Being under pressure, Rarymond Poincaré, the then president of the French Republic, ended up ordaining the creation of the army dentist within the French Military Health Service. Hence, considering such an achievement, who were the major actors who gave dental surgery such an impetus? What were the elements that stood in its way? showed numerous acts of bravery and heroism

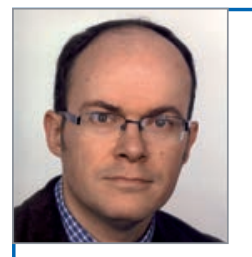

- Xavier RIAUD, Docteur en chirurgie dentaire, Docteur en épistémologie, histoire des sciences et des techniques, Lauréat et membre associé national de l'Académie nationale de chirurgie dentaire, Membre libre de l'Académie nationale de chirurgie. 145, route de Vannes, 44800 Saint-Herblain xavier.riaud@wanadoo.fr 


\section{État des lieux de la dentisterie à la fin du $X X^{e}$ siècle et au début du XX ${ }^{\mathrm{e}}$ siècle}

Au cours du XIX ${ }^{\mathrm{e}}$ siècle, la chirurgie dentaire connaît des avancées fulgurantes. Ainsi, aux États-Unis, en 1839 , la première école dentaire ouvre-t-elle ses portes à Baltimore, les cours étant délivrés dans une église jusqu'en 1840. En 1844, Horace Wells, dentiste à Hartford, demande à son associé,John Riggs, de lui enlever une dent de sagesse douloureuse, après avoir inhalé du protoxyde d'azote. Lopération connaît un franc succès. C'est la première utilisation du protoxyde d'azote à des fins thérapeutiques sur l'homme. En 1846, c'est au tour de Thomas Morton, autre dentiste et ancien associé de Wells, d'enlever une dent à un marchand de Boston après avoir utilisé de l'éther sulfurique pour anesthésier le malade. Là encore, l'intervention se déroule sans rencontrer de problème. En France, en 1827, l'arrêt Delpeuch rend le droit aux femmes d'exercer l'art dentaire. Le droit de faire de la chirurgie leur avait été enlevé sous Charles VIII en 1484. En 1880, l'École dentaire de Paris, première du genre en France, ouvre ses portes. C'est au tour de l’École odontotechnique toujours dans la capitale de délivrer ses premiers enseignements en 1884. En 1892, la loi Brouardel confère ses lettres de noblesse à la dentisterie en lui donnant de vrais statuts. Du nom d'un grand médecin, Paul Brouardel (1837-1906), il faut avoir un diplôme pour avoir le droit d'exercer sur le sol français, obtenu après avoir suivi une formation reçue au sein d'une faculté de médecine et sanctionnée à la fin par un examen. En 1897, suite à l'incendie du Bazar de la Charité, une première identification en odontologie médico-légale, en l’occurrence celle de la duchesse d'Alençon, sœur de Sissi, impératrice d'Autriche, est avalisée par la justice. En 1900, la Fédération dentaire nationale regroupe tous les dentistes au sein d'une même association [13]. C'est donc, à l'entame de la Grande Guerre, une profession balbutiante qui s'engage dans le conflit.

\section{Quid du dentiste au sein de l'armée avant la guerre?}

Au début du $\mathrm{xx}^{\mathrm{e}}$ siècle, le dentiste effectue son service militaire au grade de soldat de $2^{\mathrm{e}}$ classe. Il n'exerce jamais son art. En 1900, deux services dentaires sont ouverts à l'hôpital du Val-de-Grâce, à Paris, et à l'hôpital Desgenettes, à Lyon. Les meilleurs élèves des écoles dentaires sont affectés à ces deux hôpitaux, le temps de leur conscription au grade de soldat de $2^{\mathrm{e}}$ classe. Les soins réalisés sont très basiques : détartrages, extractions et obturations dentaires. Le 10 octobre 1907, la circulaire n 60 du ministre de la Guerre vise à organiser un service de stomatologie dans l'armée. L'état bucco-dentaire du soldat préoccupe. Un examen dentaire est effectué à l'incorporation de chaque soldat et est répertorié sur des registres [17]. La circulaire du ministre de la Guerre du 2 décembre 1910 ordonne la création de trois cabinets dentaires de garnison (Paris, Lyon et Bordeaux). Des soins basiques y sont là aussi prodigués. Le dentiste est sous l'autorité du médecin en chef de la place. En 1913, la Fédération dentaire nationale fait la proposition d'un corps de dentistes au sein de l'armée. Le président du Conseil Alexandre Millerand refuse cette motion. Le 5 mai, le refus est confirmé en attestant qu'en cas de conflit, le dentiste ne constituerait que « gêne et embarras ». Le 12 juillet, le même confirme un refus systématique à toute nouvelle proposition [2].

\section{La guerre éclate en 1914}

Un service dentaire est mis en place dans l'infanterie coloniale dès les premiers échanges de tirs. Au début de la guerre, pour le reste de l'armée, les dentistes noccupent que des fonctions subalternes (brancardier, infirmier, simples soldats, etc.). Ils se distinguent par leur bravoure, souvent au prix de leur vie. Jean Piel Melcion d'Arc, appartenant à un régiment de marche de zouaves, «s'est fait tuer héroïquement le 13 novembre 1914, en repoussant une attaque allemande qui tentait de franchir un pont (bataille de l'Yser). » Il est porté à l'ordre du régiment [17].

\section{Une hygiène dentaire calamiteuse}

Pendant tout le $\mathrm{XIX}^{\mathrm{e}}$ siècle, il est communément admis qu'un soldat dépourvu de ses incisives centrales ne peut déchirer les cartouches de poudre qui permettent de charger son fusil par la gueule. Aussi est-il soit exempté de service militaire, soit affecté à l'artillerie ou à des tâches manutentionnaires. Cet état au début de la guerre entraîne l'inaptitude [2].L'hygiène du soldat est inexistante. Les blessures de la face à traiter sont très délabrantes. L'alimentation est déplorable (tabac à priser, alimentation carnée, boîtes de conserve, alcool, etc.), provoquant l'apparition de gingivites, de parodontites, de caries, de pulpites ou encore d'abcès. L'absence de dents est responsable de troubles phonétiques chez les officiers, posant de gros problèmes dans le commandement. Les soldats n'ont jamais été suivis, ne sont pas informés des moyens de prévention et d'hygiène dentaire. Devant les inaptitudes, les délais effarants pour se faire soigner privant le front de nombreux soldats, trop nombreux au goût du commandement, une prise de conscience se fait indubitablement. Il faut les soigner de leurs maux afin qu'ils rejoignent rapidement les combats. Chaque homme compte. Le commandement va s'employer à remédier à ce problème [17]. Les dentistes soignent, mais délivrent aussi des préceptes d'hygiène bucco-dentaires. 

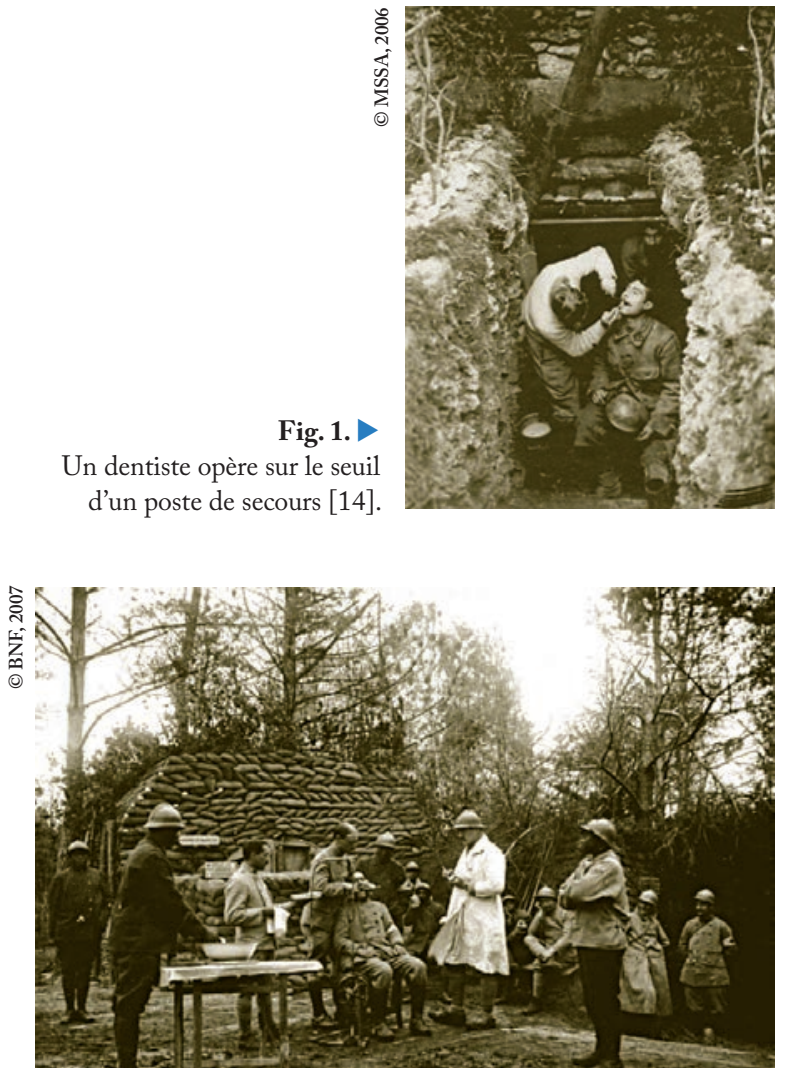

$\Delta$ Fig. 2.

Dentiste au front, près du Chemin des Dames - septembre 1917 [3].
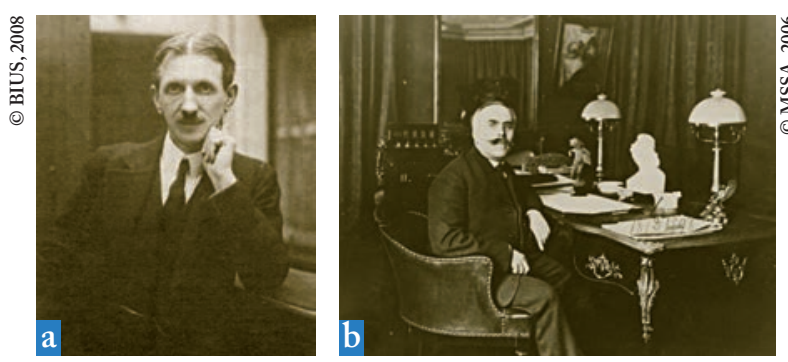

$\Delta$ Fig. 3.

a. Georges Villain (1881-1938) [4, 20].

b. Justin Godart (1871-1956) [14].

\section{Premières mesures...}

Le 15 octobre 1914, une circulaire ministérielle permet que les dentistes soient incorporés dans les sections d'infirmiers militaires afin d'y exercer leur métier pour les soins d'urgence aux combattants, mais toujours en tant que soldats. Le même jour, le premier cabinet dentaire de campagne voit le jour à Clermont-enArgonne, sous la direction du médecin aide-major de $1^{\text {re }}$ classe, Armand Lévy. Le 10 novembre, une circulaire décrète l'ouverture de trois centres de stomatologie et de prothèse maxillo-faciale à Paris, Lyon et Bordeaux, celui du Val-de-Grâce étant le premier car existant avant la guerre [10]. Le 21 décembre, un dentiste prothésiste, recruté dans les formations sanitaires ou les corps de troupe, est affecté dans les hôpitaux d'évacuation. Sa mission consiste à appliquer des pansements et des appareils provisoires de contention aux blessés atteints de mutilations de la face et des mâchoires. Il est préconisé qu'à l'avant, des dentistes qualifiés donnent aux soldats les soins nécessités par des affections dentaires. Le 24 décembre, une nouvelle circulaire autorise les «Directeurs régionaux du service de santé à faire appel aux concours bénévoles pour assurer le fonctionnement des cabinets dentaires de garnison là où ils ne trouveront pas de dentistes mobilisés " [1,7]. Le 10 mars 1915, le Journal Officiel publie les décisions de la Commission supérieure consultative du service de santé, mais ses décisions ne sont pas appliquées tout de suite. Un dentiste est affecté dans chaque régiment au service dentaire. Une automobile dentaire, composée d'un dentiste et d'un technicien dentaire, est fournie à chaque corps de troupe [15]. Les dentistes sans affectation font partie du contingent des infirmiers militaires. Le 14 avril, le ministre de la Guerre, Alexandre Millerand, accepte, par lettre officielle, la coopération de l'École dentaire de Paris pour les soins à donner aux militaires. Le 10 mai, celle du Comité de secours pour les blessés des maxillaires et de la face est approuvée par le même ministre. Le 11 juin, lécole doit assurer le service d'une ambulance de 200 lits pour les mutilés de la face, créée à l'hôpital des convalescents du lycée Michelet de Vanves. Ce service est pourvu en personnel et en matériel provenant de l'école [6]. Le $1^{\text {er }}$ juillet, Justin Godart devient sous-secrétaire d'État au Service de santé. Le 31 juillet, il visite la première automobile dentaire. Le même jour, Godart émet un arrêté qui décide l'appareillage des édentés en 15 à 20 jours. Le 25 août, il visite l'hôpital du lycée Michelet à Vanves. Confronté à l'abnégation de ces hommes, des journaux s'émeuvent de l'absence des dentistes au sein des équipes de stomatologie (Petit Journal 20/08/1915, France de demain 21/08/1915 et L'Humanité 29/08/1915). Le 31 août, Godart convoque, à son bureau, la direction de l'École dentaire de Paris et lui demande la rédaction d'un rapport pour la création d'un service dentaire et la fonction de dentiste militaire. Ce texte, rédigé par Georges Villain, lui est remis le 9 septembre 1915. Le 13 septembre, Godart visite cette école $[1,6]$.

\section{6 : une année cruciale...}

Le 26 février 1916, le ministre de la Guerre Joseph Galliéni demande la création d'un corps de dentiste militaire dans l'armée de terre au président de la République Raymond Poincaré. Le jour même, dans un décret en date du 26 février paru au Journal Officiel du 3 mars, Poincaré crée un corps de dentistes militaires pour l'armée de terre et pour la durée de la guerre seulement. Le 27 février, Godart précise dans une instruction qu'ils seront 1000 , avec le grade 


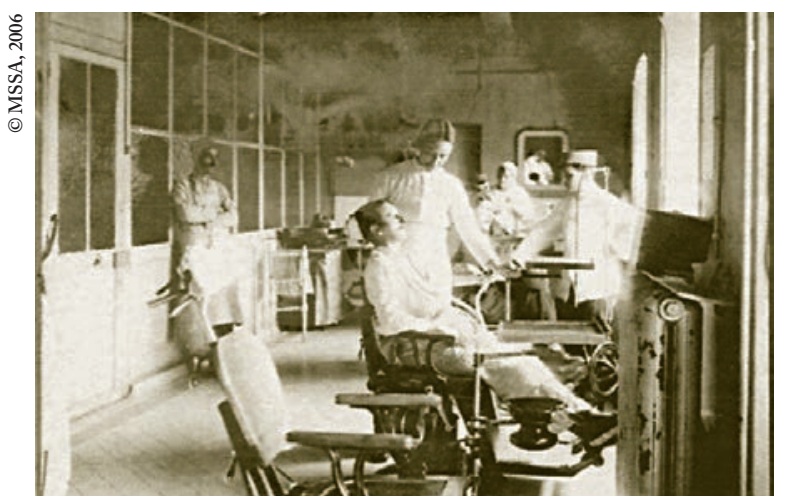

$\Delta$ Fig. 4.

Salle de clinique du service de stomatologie. Hôpital du Val-deGrâce, Paris [14].

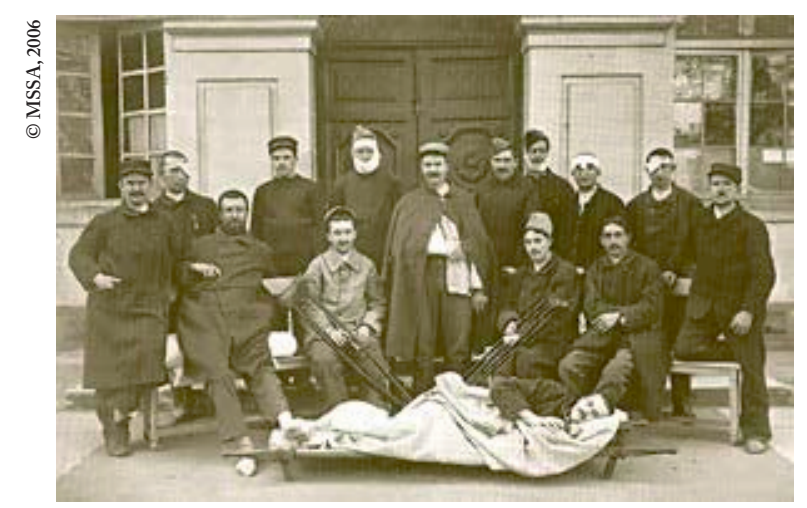

$\Delta$ Fig. 5.

Ve division des blessés de la face. Hôpital du Val-de-Grâce, Paris [14].
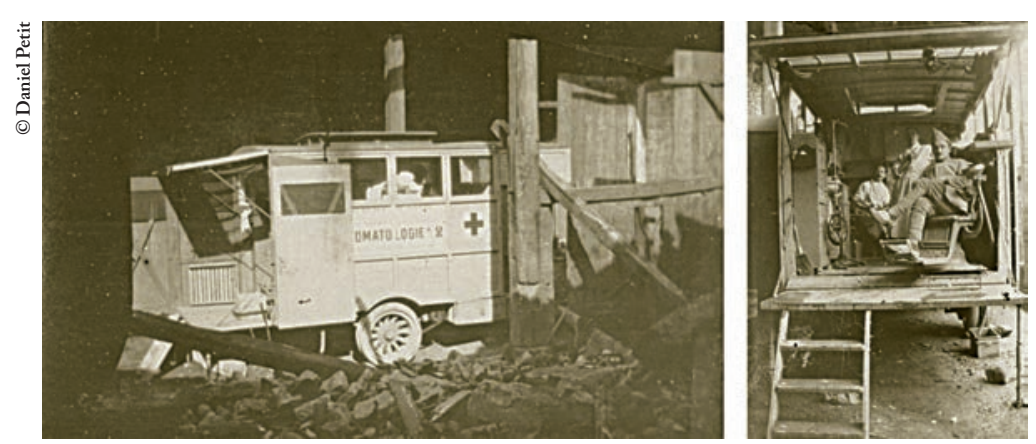

$\Delta$ Fig. 6.

Automobile dentaire française et son intérieur pendant la Grande Guerre [16].

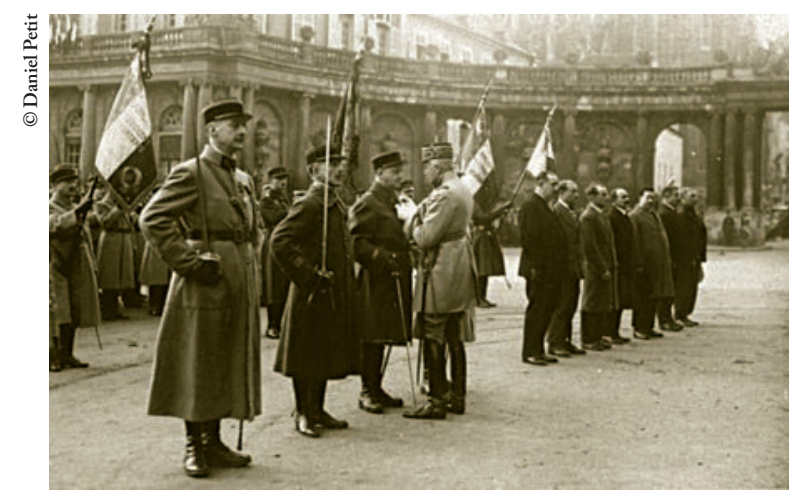

$\Delta$ Fig. 7.

Remise de la Légion d'honneur à Henri Petit (1885 - ?), dentiste, à Nancy (1930) [16]. d'adjudant. Leur tenue est celle de l'adjudant-infirmier avec un caducée argent complété de la lettre $\mathrm{D}$ haute de $1 \mathrm{~cm}$. Ils sont rattachés aux ministères des Armées et de l'Intérieur, et placés sous les ordres du médecin-chef de leur unité. Ils sont porteurs du brassard prévu par la Convention de Genève signée par les Français, le 22 septembre 1864 [18]. En janvier 1916, Blatter, président de la Fédération dentaire nationale (FDN), et Villain, son secrétaire, se rendent à plusieurs reprises auprès de l'amiral Lacaze, ministre de la Marine, pour créer un corps de dentistes militaires dans la Marine. Le $1^{\text {er }}$ mars, Lacaze envoie un rapport à Poincaré pour la création de l'emploi de « chirurgien-dentiste de la Marine » [8]. Il donne son accord immédiat. Les dentistes de la Marine sont assimilés aux médecins auxiliaires, avec la même tenue et les mêmes insignes. Dès la parution des décrets, la FDN s'empresse d'envoyer le texte à tous les dentistes français dans une lettre du 3 mars. Le 4 mars, le JO publie un décret précisant que l'amiral Lacaze est autorisé à recruter des dentistes pour seconder les médecins de la Marine sous les ordres desquels ils sont placés. Un décret à la même date ordonne que les dentistes non gradés soient affectés dans des sections d'infirmiers. Le 9 juin, l'instruction ministérielle sur les services de stomatologie, n $81193 / 7$, vraie charte des dentistes militaires, établit de façon complète et détaillée l'organisation des centres de chirurgie et de prothèse maxillo-faciale, des centres d'édentés et des cabinets dentaires de garnison $[1,6,11]$.

\section{Le Congrès dentaire interallié}

Un événement se tient à Paris en 1916 : le Congrès dentaire interallié. Ses séances du 9 au 13 novembre sont consacrées aux démonstrations et aux communications axées sur le traitement des fractures des maxillaires, et en particulier du maxillaire inférieur. Les visites dans les divers services et les différentes formations de Paris soccupant de prothèses et de restaurations maxillo-faciales ont lieu du 14 au 18. L'assemblée générale est tenue le lundi 13 novembre, à l'École dentaire de Paris, au 45 rue de la Tour d'Auvergne $[17,20]$. Une exposition complète offre aux congressistes divers moulages et appareils de contention temporaire ou définitive, des appareils de redressement, de blocage des mâchoires, de dilatation pour les divers trismus, les atrésies labiales et buccales. La séance solennelle est présidée par Justin Godart. L'organisateur est Georges Villain. Les actes du congrès sont publiés par Villain en 1917. Ils représentent 2 tomes, 1600 pages et 1100 figures. Villain les publient en moins d'un an sans négliger ses responsabilités. À l'issue du colloque, Villain est unanimement applaudi. Ce congrès connaît un succès considérable, mais est la cause d'un ralentissement certain des thérapeutiques maxillo-faciales, les praticiens ayant déserté leurs postes pour y assister [1, 6, 10]. 


\section{Autres évolutions}

Le $1^{\text {er }}$ décembre 1916, Lacaze sollicite à nouveau Poincaré pour qu'il accorde des possibilités d'avancement aux dentistes de la Marine. Le Président signe un décret en ce sens dès le 2 décembre. Les dentistes peuvent ainsi être nommés médecins de $3^{\mathrm{e}}$ classe et de $2^{\mathrm{e}}$ classe, mais leur nombre est limité au tiers de l'effectif total du personnel [2,12]. À partir de 1917, les écoles dentaires organisent des centres d'appareillage pour les malades ambulatoires, en liaison avec les hôpitaux militaires et les centres de stomatologie. Le 23 février 1917, la limitation des dentistes recevant un avancement dans la Marine est abrogée par un nouveau décret. Le 10 mars, Godart décrète la gratuité des appareils dentaires pour les soldats et les sous-officiers. Le 7 avril, le dentiste militaire régimentaire voit le jour. À la fin 1917, 50 dentistes sont recensés. Le 3 juillet, une circulaire informe les dentistes qu'ils recevront tout le matériel nécessaire à leur exercice à compter du $1^{\text {er }}$ septembre $[1$, 6]. Le 8 février 1918, Godart quitte ses fonctions, salué par la FDN. Louis Mourier (1873-1960) le remplace. Le 25 mars, un projet de loi est déposé pour la création d'officiers dentistes et est déposé à la Chambre des députés, au nom du ministre de la Guerre et du ministre des Finances. Le 20 octobre 1918, la loi du 18 octobre 1918 visant à la création d'officiers dentistes au sein du service de santé paraît au JO. Les dentistes peuvent prétendre au grade de dentiste de $2^{\mathrm{e}}$ classe (sous-lieutenant) et de $1^{\text {re }}$ classe (lieutenant). Un corps d'officiers dentistes est définitivement constitué sans limitation de durée [17]. Le décret du 11 janvier 1919 précise qu'un dentiste auxiliaire a le grade d'adjudant et ne peut devenir dentiste de $2^{\mathrm{e}}$ classe que s'il a un an d'exercice en période de guerre. De même, pour un $2^{\mathrm{e}}$ classe pouvant être promu dentiste de $1^{\text {re }}$ classe. Le nombre de dentistes auxiliaires ne doit pas dépasser 1000 , celui de $2^{\mathrm{e}}$ classe, 100 et celui de $1^{\text {re }}$ classe, 25 . Le même jour, Louis Mourier promulgue une instruction qui accompagne le décret précédent. Celle-ci stipule que les dentistes officiers sont nommés par le ministre luimême [1]. Pour cela, il faut faire état de ses diplômes, des travaux scientifiques, du temps de présence aux armées et des citations. L'uniforme des dentistes auxiliaires est celui des médecins auxiliaires et celui des $2^{2 s}$ et des $1^{\text {res }}$ classes est celui des médecins aide-majors avec le bandeau du képi. Les insignes du collet sont en velours de couleur violette, dite «prune » [2]. Un dentiste est assigné à chaque formation militaire ou médicale de l'avant (excepté 2 qui sont responsables d'une unité mobile), et dans la zone des étapes. À l'intérieur, un praticien rejoint les petites structures et leur nombre varie en fonction de l'importance des organismes hospitaliers. L'Odontologie du 30 mars 1919 annonce la nomination d'une première série d'officiers dentistes d'après la loi du 18 octobre 1918. Le JO n'annonce la nomination de 50 dentistes auxiliaires au grade de dentiste de $2^{\mathrm{e}}$ classe de réserve que le 8 juin 1919 [17]. De 1914 à 1918, 88 dentistes meurent sur le front. 156 citations leur sont délivrées $[1,6]$.

\section{La Fédération nationale des chirurgiens- dentistes de réserve (FNCDR)}

En 1925, les premiers officiers dentistes rentrés dans la vie civile se regroupent pour former l'Amicale des dentistes des armées de terre, de mer et de l'air de la région militaire de Paris, lors du $3^{e}$ Congrès de médecine et de pharmacie militaire. Elle est présidée par Jacques Filderman [18], héros de la guerre. L'objectif est de garder les liens tissés entre eux et de s'entraider afin de reprendre une vie normale [9]. Une préparation militaire spéciale supérieure facultative (PMS) d'une durée de deux ans est créée, permettant aux étudiants en chirurgie dentaire, une fois cette PMS obtenue, d'accomplir la dernière partie de leur service militaire en qualité de dentiste militaire de $2^{\mathrm{e}}$ classe, l'équivalent du grade de sous-lieutenant. Ils intègrent des cabinets dentaires de garnison, des centres dédentés ou encore des services de prothèse maxillo-faciale. En 1927, ces centres sont au nombre de huit. Les étudiants n'ayant pas accompli leur PMS ne peuvent intégrer que le service des infirmiers. Les dentistes se regroupent dans des amicales dans chaque région où ils demandent au ministre de la Guerre la permission d'y ouvrir une école de perfectionnement des chirurgiens-dentistes (EPOR). La Direction centrale du Service de santé des armées (DCSSA), se préoccupant de varier les branches du service de santé, accorde son soutien, ce qui débouche à l'ouverture de la première EPOR. Cette école offre ses premiers services lors de cours mensuels à l'hôpital militaire Villemin en octobre 1926. Lexemple donné par Paris sétend à partir de 1931 et chaque région militaire peut jouir de son EPOR. Intégrer cette école compte vite pour l'avancement en grade [10]. Toutes ces amicales régionales se regroupent en une Fédération nationale des chirurgiens-dentistes de réserve, en 1933. La DCSSA organise la formation des dentistes à toutes sortes de fonctions : anesthésiste, aide-chirurgien, radiologue, secouriste, etc. Au Valde-Grâce, de 1931 à 1933, Gustave Ginestet, alors $1^{\text {er }}$ assistant de chirurgie des hôpitaux militaires, forme des dentistes à la chirurgie maxillo-faciale [5]. Entre 1930 et 1940, des cabinets dentaires sont mis en place dans la Marine, sur les cuirassés Paris, Provence et le porte-avions Béarn [2].

\section{Les dentistes d'active}

Le service de santé des armées décide de mettre en place des structures médicales mobiles pouvant suivre un régiment ou une division, plus adaptées aux 
schémas des guerres dites de mouvement. Ainsi, des groupes chirurgicaux mobiles, des hôpitaux avancés, des hôpitaux médicaux d'évacuation et divers services de santé font leur apparition. Dans chacun d'eux, des places pour les dentistes sont pourvues. La loi du $1^{\text {er }}$ avril 1923 prévoit ainsi, dans l'article 39, que les étudiants en dentaire doivent accomplir leur service militaire dans le service de santé comme infirmier s'ils n'ont pas fini leur cursus dentaire, ou en tant que dentiste de $2^{\mathrm{e}}$ classe s'ils sont diplômés [9]. Le décret du 7 juillet 1929 stipule qu'un corps d'officiers de réserve, avec parmi eux les dentistes, est instauré au sein du service de santé en cas de mobilisation. Il précise que le dentiste de $2^{\mathrm{e}}$ classe peut devenir dentiste de $1^{\text {re }}$ classe au bout de quatre années de service. Le décret du 4 avril 1934 dote la marine de 50 postes et la loi du 19 décembre 1934 permet au dentiste de réserve d'accéder au grade de capitaine [2,5].

\section{La mobilisation}

La Seconde Guerre mondiale éclate le 3 septembre 1939. S'en suit une mobilisation générale en France. Les dentistes de réserve sont mutés, la plupart en tant que lieutenants, vers diverses zones stratégiques. Leur affectation se fait soit dans les formations sanitaires de divisions, soit dans les ambulances chirurgicales ou dans les régiments de l'avant. Leur travail concerne peu le domaine dentaire. Ils servent comme assistants pour des soins médicaux. Seuls, quelques-uns exercent dans des cabinets dentaires de garnison ou d'hôpitaux. En effet, le service de santé a créé un cabinet dentaire dans chaque chef-lieu de secteur et dans les garnisons les plus importantes. Mais, en première ligne, ils interviennent dans les postes de secours pour soigner les blessés. En zone divisionnaire, ils collaborent avec des médecins et chirurgiens au sein des groupes chirurgicaux mobiles ou avancés. À l'arrière, ils sont employés par les centres spécialisés en chirurgie maxillo-faciale ou encore dans les services de radiologie. En ce début de guerre, les premiers soins aux blessés sont prioritaires et demandent beaucoup d'efforts. D'autant plus qu'aux militaires, s'ajoutent les blessés civils, victimes des bombardements $[2,5,12]$. Pour ceux ayant suivi l'enseignement délivré dans les EPOR, les affectations des dentistes se font aisément dans les services chirurgicaux [10]. Afin de garder une trace écrite, une fiche schématique est établie pour chaque soldat et un journal est tenu quotidiennement par le chef de service du cabinet dentaire. $Y$ sont inscrites toutes les consultations et interventions réalisées. Des remises en état de la cavité buccale sont effectuées avant toute opération militaire, avec un détartrage préliminaire obligatoire, première démarche d'hygiène. Des extractions sont faites en grand nombre. Toute attribution d'un appareil prothétique fait l'objet au préalable d'une demande auprès du directeur du service de santé de la région par l'intermédiaire du stomatologiste principal pour accord. Les extractions étant faites avant la réalisation d'un éventuel appareil ne le sont qu'une fois l'avis favorable délivré au chef de service du cabinet dentaire. Un mois et demi après, l'appareil est conçu. Si un refus motivé revient, seules les extractions sont faites [10].Dans chaque cabinet dentaire, le personnel est composé d'un chef de service, qui est un médecin stomatologiste ou un dentiste militaire, et de dentistes militaires pour l'assister selon les besoins, nommés par le stomatologue principal de la région. Ce chef de service est sous la direction du médecin-chef de la formation à laquelle il est rattaché telle que le corps de troupe, l'infirmerie régimentaire,l'hôpital militaire, etc., tout en demeurant sous les ordres du stomatologue principal [9]. En 1940, la formation continue est assurée par des revues comme l'Odontologie qui cesse sa parution entre juin/juillet 1944 et mars/avril 1945, faute de publicité pour la promouvoir.

\section{Le service de stomatologie}

Après la capitulation des Français, le 22 juin 1940, signée à Rethondes, de nombreux chirurgiens-dentistes démobilisés sont rappelés pour soigner les blessés de la face. À côté des organisations secondaires que sont les cabinets dentaires de garnison qui fonctionnent dans les chefs-lieux de secteur et dans les garnisons les plus importantes, il existe des services de stomatologie. Ils sont mis en place dès le début de la guerre.Il y a ainsi deux articulations différentes concernant ces structures à l'arrière. Ce sont les services techniques inter-régionaux et les services techniques régionaux. 11. Le centre inter-régional de chirurgie et de prothèse maxillo-faciale est indépendant du service régional, mais demeure sous les ordres du directeur du service de santé de la région dont il dépend. Un chirurgien, chef de service, $y$ est à sa tête et dispose comme adjoint d'un médecin stomatologiste qui, lui, est chef du service de prothèse maxillo-faciale. Ces deux professionnels ont pour équipe tous les spécialistes qui leur sont nécessaires pour la chirurgie et la prothèse : stomatologues, chirurgiens-dentistes et techniciens en prothèse dentaire. Y sont traités principalement les délabrements osseux des maxillaires, de la face et du cou grâce à cette formation mixte chirurgicale et stomatologique. Au contraire, les fractures plus simples des maxillaires, c'est-àdire sans perte de substance, sont soignées dans les centres de stomatologie régionaux. De même, les édentés simples ne sont pas admis. Il existe pour ces blessés des centres d'édentés où ils peuvent être appareillés. Chaque centre est bien spécialisé. Le tout est de bien orienter, et rapidement, les blessés. 12. Dans une région, il n'existe qu'un seul service de stomatologie, voire plusieurs, si la région est très étendue. Un seul centre de prothèse dentaire pour 
édentés travaille à la confection des appareils dentaires de tous les édentés venus dans les cabinets dentaires des garnisons de la région. Ces services de stomatologie et de prothèse dentaire régionaux comportent 50 à 100 lits sur 10000 à 20000 lits pour tout le milieu médical. Les affections odonto-stomatologiques trop importantes pour être traitées dans les cabinets dentaires, les affections dentaires, les fractures simples des maxillaires et leur appareillage, et les extractions dites chirurgicales y sont opérées. L'appareillage prothétique simple des édentés y est effectué. La direction de ces centres régionaux est assurée par le stomatologue principal de la région.Il doit surveiller le centre de stomatologie et de prothèse dentaire, et les cabinets dentaires. Son effectif comprend un technicien en prothèse dentaire capable de faire un appareil par jour, soit 30 appareils par mois, et un dentiste militaire capable de fournir du travail à 4 ou 5 techniciens. Un officier s'occupe de la gestion du matériel comprenant l'entretien et les demandes. Il tient également un registre sur ce qui a été posé « en bouche », avec la description de l'appareil. Cela permet par la suite de justifier du prix de chaque appareil. Enfin, le directeur de la région peut ainsi suivre l'activité du centre d'édentés grâce à un compte rendu mensuel $[5,10]$. Depuis l'instruction sur l'aptitude au service militaire du 7 décembre 1938, pour les troupes métropolitaines, suivant un calcul donnant le coefficient de mastication du militaire, en cas de déficience de l'état général du militaire attribuable à l'insuffisance de la denture, l'appareillage est rendu possible, mais seulement lorsque son coefficient de mastication est inférieur à $25 \%$. Ainsi, tout homme, même complètement édenté, mais muni d'un appareil de prothèse complet, peut être gardé dans le service [10]. Dans la phase maxillo-faciale d'urgence, le blessé doit être conduit à un centre maxillo-facial entre 2 et 10 jours après la blessure. Plus le traitement débute rapidement et plus les chances de succès sont importantes. Le 11 novembre 1942, l'armée allemande envahit la zone libre. Le mois suivant, l'armée d'armistice est dissoute. Dès lors, le service de santé de l'armée française n'existe plus. Le soutien médical est confié pour toute la durée du conflit aux Britanniques et aux Américains $[9,10]$. Nombre de dentistes démobilisés en 1940 entrent alors en Résistance, leurs cabinets dentaires servant de plaques tournantes pour l'échange d'informations. Beaucoup d'entre eux meurent fusillés par la Gestapo. D'autres sont déportés et en reviennent diminués. D'autres s'engagent dans les forces françaises libres [18].

\section{L'après-guerre}

Sous l'impulsion de la Fédération des amicales des dentistes de réserve, du médecin et lieutenant colonel
Ginestet, un projet de création de 50 postes de dentistes de carrière est abandonné, faute d'argent. Dans le même temps, la guerre d'Indochine débute en 1945. Le Parlement ne donne pas son agrément à l'envoi du contingent. Ce sont donc des dentistes volontaires civils, engagés dans le Corps auxiliaire des forces armées en Extrême-Orient (CAFAEO), qui partent. Trentehuit cabinets dentaires et sept véhicules dentaires officient sur le front et délivrent 18000 consultations, et 5800 prothèses. Cette action contribue largement à maintenir apte au combat l'équivalent de plusieurs bataillons $[2,5]$.

Lorsque la guerre d'Algérie éclate, cette fois, le contingent est envoyé. Le nombre de dentistes oscille entre 8 au début du conflit (1954) et 185 à la fin (1962). Certains d'entre eux font un séjour de 29 mois en Algérie. Pourtant, aucune avancée sensible n'est apparue sur cette période.

En 1965, Jean Jardiné, président de la CNSD, réclame la création d'un corps de dentistes d'active, affirmant que la France est le seul pays de la CEE à ne pas en disposer. Pierre Mesmer, alors ministre aux Armées, s'y oppose, estimant que ceux de réserve suffisent [2].

\section{En France}

En 1948, la Direction centrale du service de santé (DCSSA) est créée. Les dentistes de réserve des trois armées passent sous l'autorité d'un seul organisme. En 1951, la loi du 24 mai et le décret du 27 juin leur conferrent le grade de commandant. Ils doivent suivre une formation d'EOR (élève officier de réserve), au Val-de-Grâce notamment, au Centre national du service de santé des armées (CNIEORSSA). Suite à un concours, en fonction du rang, ils peuvent choisir leur affectation. En 1960, un autre centre est ouvert à Libourne qui devient une école. Il formera tous les dentistes appelés jusqu'en 2001. Le décret du 4 juin 1971 donne un vrai statut aux dentistes puisqu'ils obtiennent les grades et l'avancement des médecins, et pharmaciens, avec pour plus haut rang, celui de colonel. La loi du 13 juillet 1972 insiste sur les modalités relatives à l'engagement des officiers de réserve en situation d'activité (ORSA), ce qui concerne les dentistes au premier plan. Un ORSA est payé en fin de contrat et peut intégrer un corps d'officiers de carrière. En novembre 1973, un premier dentiste qualifié ORSA est affecté en corps de troupe [2,5]. Son contrat n'excède pas huit ans. Il peut être réitéré, mais ne dépasse pas vingt ans. Le décret du 17 mai 1974 réforme les grades des chirurgiens-dentistes de réserve : chirurgien-dentiste en chef (colonel et lieutenant-colonel), chirurgien-dentiste principal (commandant) et chirurgien-dentiste (capitaine) [2,5]. À partir de 1977, l'armée n'appelle plus que des ORSA. Pourtant, ils ne sont qu'au nombre de cinq dans les hôpitaux militaires en 1986, pour 327 dentistes aspirants dans les corps de 
troupe. Mais leur nombre reste insuffisant et l'essentiel des soins est dispensé par des stomatologues militaires et des appelés.

\section{La guerre du Golfe}

En 1990, les affrontements démarrent dans le Golfe persique. Le 3 mars 1991, des dentistes ORSA sont envoyés en zone arrière dans des bâtiments de la marine nationale. Sur le TCD Foudre, le dentiste soigne 458 soldats en 633 séances. Sur le front, ce sont les stomatologues qui officient. À l'hôpital de campagne Daguet, le médecin effectue 482 consultations entre le 20 janvier et le 8 mars 1991 [2, 5, 12]. En mars 1991, une mission sanitaire est réalisée par deux praticiens auprès des populations kurdes.

\section{Nouveaux décrets}

Suite au décret du 28 juillet 1978 confirmé par l'arrêté du 2 septembre 1991, la DCSSA engage deux dentistes sous contrat pour une durée de quatre ans, avec possibilité de prolongation dans la limite des dix ans. Les dentistes sont alors présents sur tous les fronts (Tchad, Liban, ex-Yougoslavie, etc.). Les besoins en soins ne font qu'augmenter et démontrent l'importance des soins dentaires qui se multiplient, malgré une aptitude pour les missions toute relative. En 1993, il y a 17 ORSA dont une femme, pour 280 du contingent. La féminisation de la profession provoque une diminution du nombre des appelés significative.

\section{Décret $\mathrm{du} 1^{\mathrm{er}}$ mars 2000}

En février 1996, Jacques Chirac annonce la professionnalisation des armées et la suspension du service national, qui est instaurée par la loi du 2 juillet 1996. Le service national est suspendu officiellement en 2002, ce qui libère un budget conséquent. Ainsi, le décret du $1^{\text {er }}$ mars 2000 permet la création d'un corps de chirurgiens-dentistes des armées. Leur rôle est d'assurer les soins dentaires, la prévention, l'hygiène et l'expertise bucco-dentaire $[2,5,12]$. Le grade de général est mis en place. Le recrutement s'effectue par concours auprès des officiers de carrière du service de santé des armées ayant assuré au moins deux ans de service actif.En juin 2000, deux décrets suppriment le qualificatif d'ORSA, mais ses modalités demeurent. Les dentistes ORSA sont automatiquement intégrés en tant qu'officier sous contrat (OSC). En 2003, ils sont au nombre de 43 dont 3 femmes. Il est prévu qu'ils passent à 58 pour les années suivantes $[2,5,12]$.

Avec tous mes remerciements au Dr Hubert Ouvrard pour son amical soutien et ses précieux conseils.

\section{Bibliographie}

[1] Augier S. Les chirurgiens-dentistes français aux armées pendant la Première Guerre mondiale (1914-1918) : Organisation d'un service dentaire et stomatologique. Lyon I, Thèse Doct. Chir. Dent., 1986.

[2] Benmansour A. « Cent ans d'histoire des chirurgiens-dentistes militaires français ", Revue historique des Armées 2003;231:106-119.

[3] Bibliothèque nationale de France, communication personnelle, Paris, 2007.

[4] Bibliothèque interuniversitaire de Santé, communication personnelle, Paris, 2008.

[5] Bourguignon P. La place du chirurgiendentiste au sein des forces armées hier et aujourd'hui : rôle de la réserve et du réserviste, Thèse Doct. Chir. Dent., Paris V, 2004.

[6] Caliot V. Rôle des chirurgiens-dentistes français aux armées durant la Première Guerre mondiale (1914-1918), Bordeaux II, Thèse Doct. Chir. Dent., 1993.
[7] Delaporte S. Gueules cassées de la Grande Guerre. Paris : Agnès Viénot (éd.), 2004.

[8] Ferret-Dussart K. La chirurgie maxillofaciale à travers l'histoire. Paris : Glyphe et Biotem (éd.), Coll. Société, histoire et médecine, 2004.

[9] Jamin S. Le chirurgien-dentiste français pendant la Seconde Guerre mondiale. Thèse Doct. Chir. Dent., Rennes, 2011.

[10] Konieczny B. Le chirurgien-dentiste dans le service de santé des armées françaises durant les guerres modernes. Nantes, Thèse Doct. Chir. Dent., 1992.

[11] Larcan A, Ferrandis J.-J. Le service de santé aux armées pendant la Première Guerre mondiale. Paris : LBM (éd.), 2008.

[12] Lecomte O,Tristan D. « Les praticiens des armées dans l'histoire de l'art dentaire ". Médecine et armées, 2010;38(5):469-476.

[13] Morgenstern H. Les dentistes français au XIX ${ }^{\mathrm{e}}$ siècle. Paris : L'Harmattan (éd.), Coll. Médecine à travers les siècles, 2009.
[14] Musée du service de santé des armées au Val-de-Grâce, communication personnelle, Paris, 2006.

[15] CEuvre collective. Science et dévouement. Le service de santé - La Croix-Rouge - Les œuvres de solidarité de guerre et d'après-guerre. Paris : Quillet (éd.), 1918.

[16] Petit D. Coll. privée de Petit Henri, communication personnelle, Nancy, 2006.

[17] Riaud X. Première Guerre mondiale et stomatologie : des praticiens d'exception. Paris : L'Harmattan (éd.), Coll. Médecine à travers les siècles, 2008.

[18] Riaud X. Pionniers de la chirurgie maxillofaciale (1914-1918). Paris : L'Harmattan (éd.), Coll. Médecine à travers les siècles, 2010.

[19] Riaud X. Communication personnelle, Saint Herblain, 2012.

[20] Mme Villain Georges. Georges Villain (1881-1938), In Memoriam, Paris, n $864 / 1000,1947$. 